\title{
Effects of fluid shear stress on the expression of Omi/HtrA2 in human umbilical vein endothelial cells
}

\author{
LIANG-LIANG SUN, LE ZHANG，XIANG-LAN MENG，FENG ZHANG，YUN ZHAO and XIN JIN \\ Department of Pharmacology, School of Medicine, Xiamen University, Xiamen, Fujian 361005, P.R. China
}

Received March 10, 2012; Accepted July 23, 2012

DOI: $10.3892 / \mathrm{mmr} .2012 .1137$

\begin{abstract}
To investigate the molecular mechanisms of laminar shear stress on the inhibition of apoptosis in endothelial cells, human umbilical vein endothelial cells (HUVECs) were starved in medium containing $2 \%$ fetal bovine serum (FBS) and treated with 15 dyne $/ \mathrm{cm}^{2}$ shear stress. We confirmed that 15 dyne $/ \mathrm{cm}^{2}$ shear stress inhibited the expression of Omi/HtrA2 at the mRNA and protein levels in cultured HUVECs. Furthermore, the release of Omi/HtrA2 from the mitochondria was induced by removal of basic fibroblast growth factor and decrease of FBS in the medium, while shear stress inhibited its release under the same conditions. These results suggest that downregulation of Omi/HtrA2 may contribute to the potent anti-atherosclerotic effect of shear stress by preventing endothelial cells from entering apoptosis.
\end{abstract}

\section{Introduction}

Shear stress induced by blood flow may play a pivotal role in the induction or prevention of atherosclerosis by affecting endothelial functions. Previous studies have demonstrated that shear stress also inhibits apoptosis of vascular endothelial cells (ECs) $(1,2)$. In contrast, flow that has a low mean shear stress and turbulence is markedly correlated with EC dysfunction, EC apoptosis and atherosclerosis (3-5). In accordance with our previous studies $(6,7)$, we identifed that physiological shear stress inhibits EC apoptosis partly by activating human inhibitor of apoptosis protein-2 (HIAP-2) and X-linked inhibitor of apoptosis protein (XIAP). Mammalian Omi/HtrA2, a nuclearencoded mitochondrial protein, is the antagonist of inhibitor of apoptosis protein (IAP). Following apoptotic stimulation, the target sequence of Omi/HtrA2 in mitochondria is cleaved, resulting in a new apoptotic activity of the N-terminus. When mammalian Omi/HtrA2 is released into the cytoplasm, it has the ability to bind and antagonize the baculovirus IAP repeat

Correspondence to: Dr Xin Jin, Department of Pharmacology, School of Medicine, Xiamen University, 168 Da Xue Road, Si Ming, Xiamen, Fujian 361005, P.R. China

E-mail:xinjin@xmu.edu.cn

Key words: shear stress, apoptosis, Omi/HtrA2, human umbilical vein endothelial cells, atherosclerosis
(BIR) function of IAPs and releases the caspases binding to the BIR. Omi/HtrA2 also promotes the catalytic cleavage of IAPs leading to their irreversible inactivation and promoting the progression of apoptosis $(8,9)$. The catalytic activity of the Omi/ HtrA2 serine protease therefore plays a major role in induced cell death. It is noteworthy that a number of studies have demonstrated the importance of the Omi/HtrA2 pathway in different cell types (10-12). However, its role in the endothelial adaptive response to laminar shear stress has, to date, not been elucidated.

In the present study, we reveal that Omi/HtrA2 expression and function are markedly inhibited following exposure of ECs to laminar shear stress. We also analyze the role of Omi/HtrA2 signaling in the response of ECs to hemodynamic force.

\section{Materials and methods}

Cell culture. Human umbilical vein endothelial cells (HUVECs) were harvested from a human umbilical vein using $0.05 \%$ trypsin with $0.02 \%$ EDTA and suspended in Dulbecco's modified Eagle's medium (DMEM) containing 20\% fetal bovine serum (FBS), $10 \mu \mathrm{g} / \mathrm{l}$ basic fibroblast growth factor (bFGF), $100 \mathrm{U} / \mathrm{ml}$ penicillin and $100 \mu \mathrm{g} / \mathrm{ml}$ streptomycin sulfate. Cells were plated onto gelatin-coated polyester sheets $(54 \times 89 \mathrm{~mm}$; Costar-Corning, Tewksbury, MA, USA), at a seeding density of $1-5 \times 10^{6}$ per sheet. The cells were cultured until they reached confluence. To avoid phenotypic changes caused by passaging, only cells experiencing 3-7 passages were used.

HUVECs were obtained and cultured as previously described (6). Cells were plated on plastic slides or polyester sheets, and flow experiments using a parallel-plate flow chamber (Fig. 1) were performed to generate unidirectional laminar shear stress $(6,7)$. Control ECs were maintained in an incubator.

DNA fragmentation assay. The isolation of fragmented DNA was conducted as follows. Briefly, after culturing for $24 \mathrm{~h}$ and starving in medium containing $2 \% \mathrm{FBS}$, cells were treated with varying levels of shear stress for $24 \mathrm{~h}$. HUVECs were lysed and treated with RNAse A and proteinase K. The DNA fragments were precipitated with ethanol, resuspended in $50 \mu \mathrm{l}$ of TE buffer and analyzed by electrophoresis.

RT-PCR and real-time PCR. The HUVECs were incubated with $2 \%$ FBS DMEM without bFGF for $12 \mathrm{~h}$, then placed in the shear stress flow chamber. ECs were exposed to $15 \mathrm{dyne} / \mathrm{cm}^{2}$ 
of shear stress for 1, 2, 4 and $6 \mathrm{~h}$, and harvested for RT-PCR. Total RNA was extracted using TRIzol (Invitrogen Life Technologies, Carlsbad, CA, USA) according to the manufacturer's instructions. PCR primers were in accordance with published sequences as follows: Omi/HtrA2 forward, 5'-GCC GTG GTC TAT ATC GAG ATC-3'; reverse, 5'-TGA GCC GTT CGA GAT AGG G-3'; GAPDH forward 5'-GCA CCG TCA AGG CTG AGA AC-3'; reverse, 5'-TGG TGA AGA CGC CAG TGG A-3'. PCR was performed for one cycle of $2.5 \mathrm{mins}$ at $95^{\circ} \mathrm{C}$, and then run for 33 cycles at $94^{\circ} \mathrm{C}$ of $30 \mathrm{sec}, 58^{\circ} \mathrm{C}$ for $30 \mathrm{sec}$ and $72^{\circ} \mathrm{C}$ for $45 \mathrm{sec}$.

mRNA levels were also quantified by real-time PCR. The amplification mixtures $(20 \mu \mathrm{l})$ contained $0.5 \mu \mathrm{l}$ of cDNA, $0.5 \mu \mathrm{l}$ of each primer and $10 \mu 1 \mathrm{SYBR}-$ Green PCR Master mix (Applied Biosystems, Foster City, CA, USA). PCR was performed at $50^{\circ} \mathrm{C}$ for $2 \mathrm{~min}, 95^{\circ} \mathrm{C}$ for $10 \mathrm{~min}$ (for AmpliTaq Gold activation) and then run for 40 cycles at $95^{\circ} \mathrm{C}$ for $15 \mathrm{sec}$ and $60^{\circ} \mathrm{C}$ for $1 \mathrm{~min}$. The fluorescence data for the SYBR-Green dye at each cycle were collected using an ABI PRISM 7300 Sequence Detection System (Applied Biosystems). The cycle threshold values were normalized against the housekeeping gene GAPDH.

Western blot analysis. HUVECs were harvested by scraping, and the cells were pelleted by centrifugation and resuspended in lysis buffer. Proteins were separated on a $10 \%$ SDS-polyacrylamide gel and transferred onto a polyvinylidene difluoride (PVDF) membrane. Anti-Omi/HtrA2 monoclonal antibody was diluted 1:500 (R\&D Systems, Minneapolis, MN, USA). Anti $\beta$-actin antibody was diluted 1:1000. Immunoblots were detected by enhanced chemiluminescence reaction reagents and signals were quantified using the Bio-Rad system image program (Quantity One; Bio-Rad, Hercules, CA, USA).

Immunofluorescence microscopy. Cells were exposed to 15 dyne $/ \mathrm{cm}^{2}$ shear stress with 2\% FBS in DMEM for 12, 18 and $24 \mathrm{~h}$, and then $100 \mathrm{nM}$ MitoTracker Red was added for $20 \mathrm{~min}$ at $37^{\circ} \mathrm{C}$. The cells were washed three times with PBS, fixed with $4 \%$ paraformaldehyde for $20 \mathrm{~min}$, permeabilized with $0.2 \%$ Triton X-100 in PBS for $5 \mathrm{~min}$, and blocked for $1 \mathrm{~h}$ with $3 \%$ BSA at $4^{\circ} \mathrm{C}$. Cells were then incubated with anti-Omi/HtrA2 (1:200 in $3 \% \mathrm{BSA}$ ) for $2 \mathrm{~h}$ at $37^{\circ} \mathrm{C}$, washed three times with PBS, permeabilized with $0.2 \%$ Triton $\mathrm{X}-100$ again, and then incubated with FITC-goat anti-mouse IgG (1:100 in 3\% BSA) for $60 \mathrm{~min}$ at $25^{\circ} \mathrm{C}$. After three more washes with PBS, cells were mounted in 1 drop of Dapi-Fluoromount-G. Finally, the fluorescence was visualized using a laser scanning confocal microscope (Fluoview, Olympus, Japan).

Statistical analysis. Statistical analysis was performed using GraphPad Prism 5 and Bio-Rad Quantity One software. The distribution of data for each variable was assessed and variables were transformed to normalize the distribution of data, if necessary. Multiple comparisons were performed by one-way analysis of variance. $\mathrm{P} \leq 0.05$ was considered to indicate a statistically significant difference.

\section{Results}

Shear stress inhibits HUVEC apoptosis. Serum starvation of HUVECs resulted in typical characteristics of apoptotic cells,

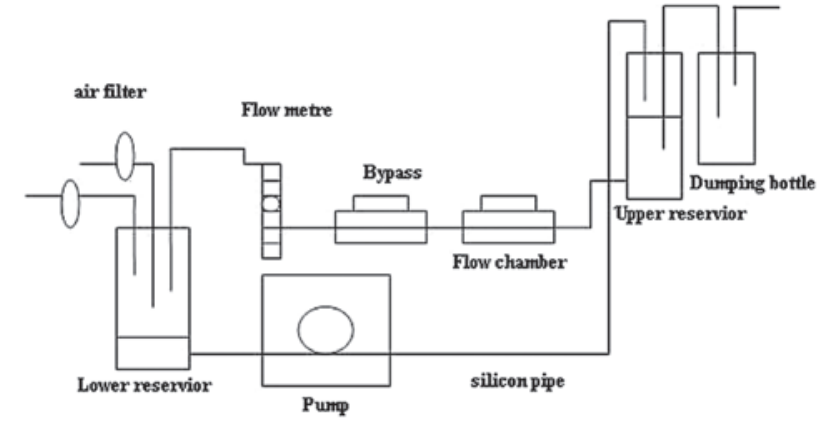

Figure 1. Diagram of flow apparatus. ECs were cultured on sheets, and then placed into the flow chamber. Different levels of shear stress were produced by adjusting the flow rate. EC, endothelial cells.

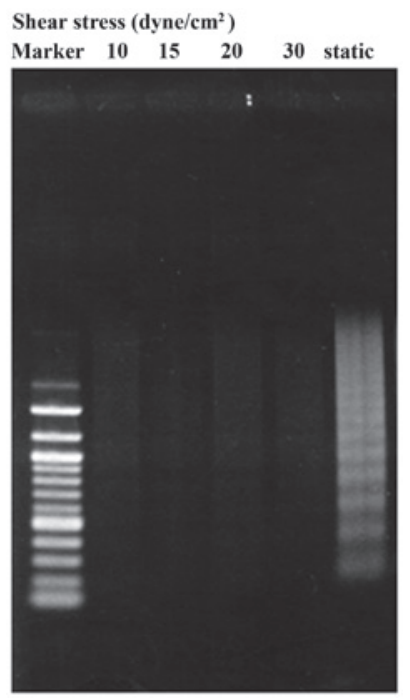

Figure 2. Inhibition of EC apoptosis by shear stress. Following exposure to shear stress for $24 \mathrm{~h}$, DNA fragmentation of HUVECs was analyzed by electrophoresis. Lane 1, molecular marker; lane 2, shear stress of $10 \mathrm{dyne} / \mathrm{cm}^{2}$; lane 3 , shear stress of $15 \mathrm{dyne} / \mathrm{cm}^{2}$; lane 4 , shear stress of 20 dyne $/ \mathrm{cm}^{2}$; lane 5 , shear stress of $30 \mathrm{dyne} / \mathrm{cm}^{2}$; lane 6 , static control ECs. EC, endothelial cell; HUVECs, human umbilical vein endothelial cells.

including shrinkage, rounding and membrane blebbing. No such effect was observed with the shear stress-treated cells. Agarose gel electrophoresis revealed a typical ladder pattern of internucleosomal DNA fragmentation in the serum-deprived cells, while the shear stress treated group demonstrated inhibition of serum starvation-stimulated apoptosis (Fig. 2).

Omi/HtrA2 expression in HUVECs. To examine whether shear stress induced expression of Omi/HtrA2 mRNA, RT-PCR and real-time PCR were performed. Before the shear stress was imposed, the HUVECs were induced to apoptosis by incubation with $2 \%$ FBS DMEM without bFGF for $12 \mathrm{~h}$. The level of mRNA expression was indicated in terms of the $\mathrm{Ct}$ value. The $\mathrm{Ct}$ values reflect the cycle number at which the fluorescence generated within a reaction crosses the threshold. $\Delta \mathrm{Ct}$ is the difference in threshold cycles for the target gene and the reference gene. In this study, GAPDH was used as the reference gene. The Omi/HtrA 2 mRNA level decreased from $4 \mathrm{~h}$ to $6 \mathrm{~h}$ following shear stress (Fig. 3A and B). 

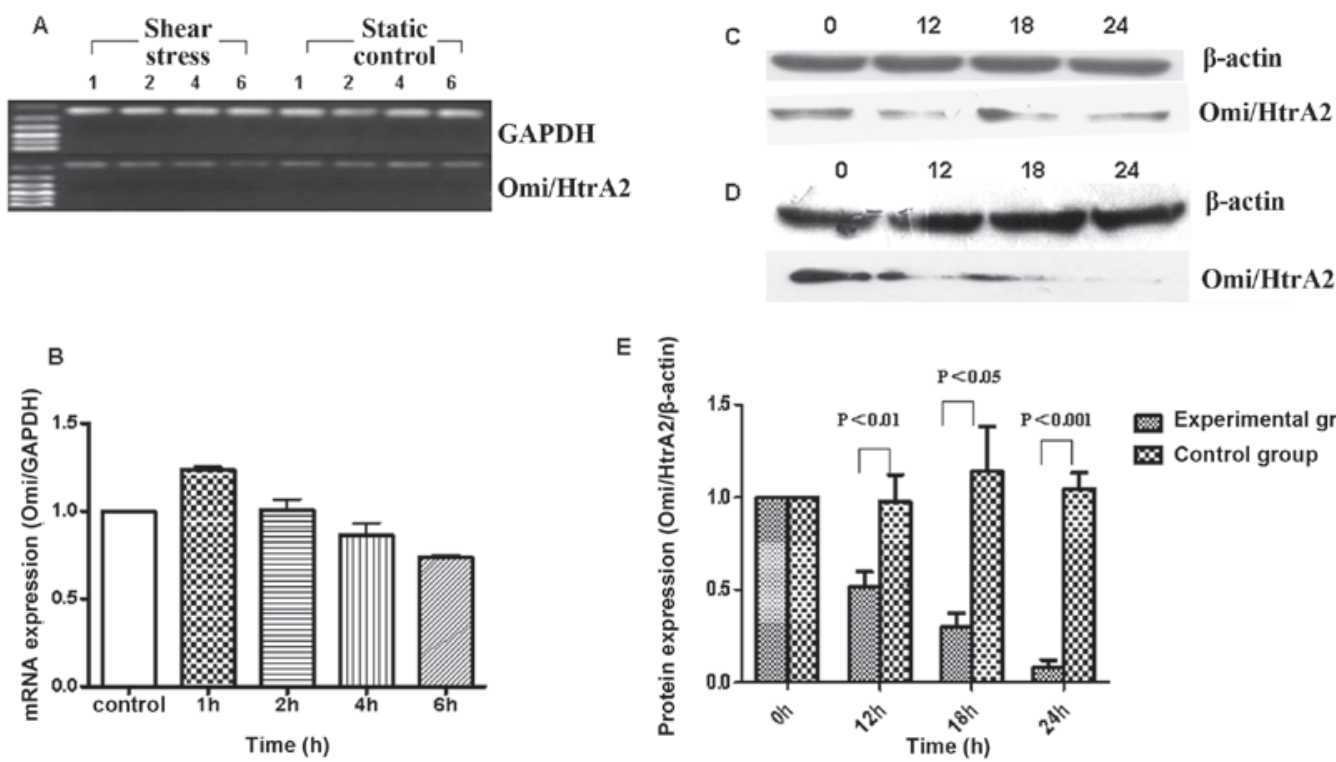
Experimental group Control group

Figure 3. Effect of shear stress on Omi/HtrA2 expression in HUVECs $\left(15\right.$ dyne/cm $\left.{ }^{2}\right)$. RNA samples were collected after $1,2,4$ and $6 \mathrm{~h}$ of incubation with or without shear stress treatment and Omi/HtrA2 expression was quantified by (A) RT-PCR and (B) real-time PCR. At $4 \mathrm{~h}$ after 15 dyne/cm ${ }^{2}$ shear stress the Omi/HtrA2 mRNA level began to drop, while the control group revealed no change. Total protein was collected after 12,18 and $24 \mathrm{~h}$ incubation with or without shear stress treatment. (C) Omi/HtrA2 total protein revealed fewer changes in the control group. (D) The level of Omi/HtrA2 protein was significantly reduced from $12 \mathrm{~h}$ to $24 \mathrm{~h}$ by 15 dyne $/ \mathrm{cm}^{2}$ of shear stress. Values represent the mean \pm SEM. This result is representative of three independent experiments. HUVECs, human umbilical vein endothelial cells.

A

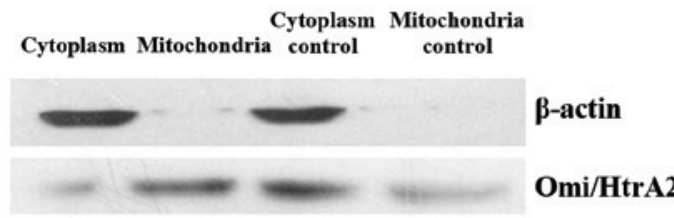

B

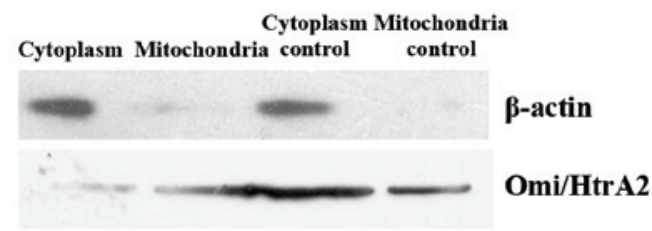

Immunofluorescence results

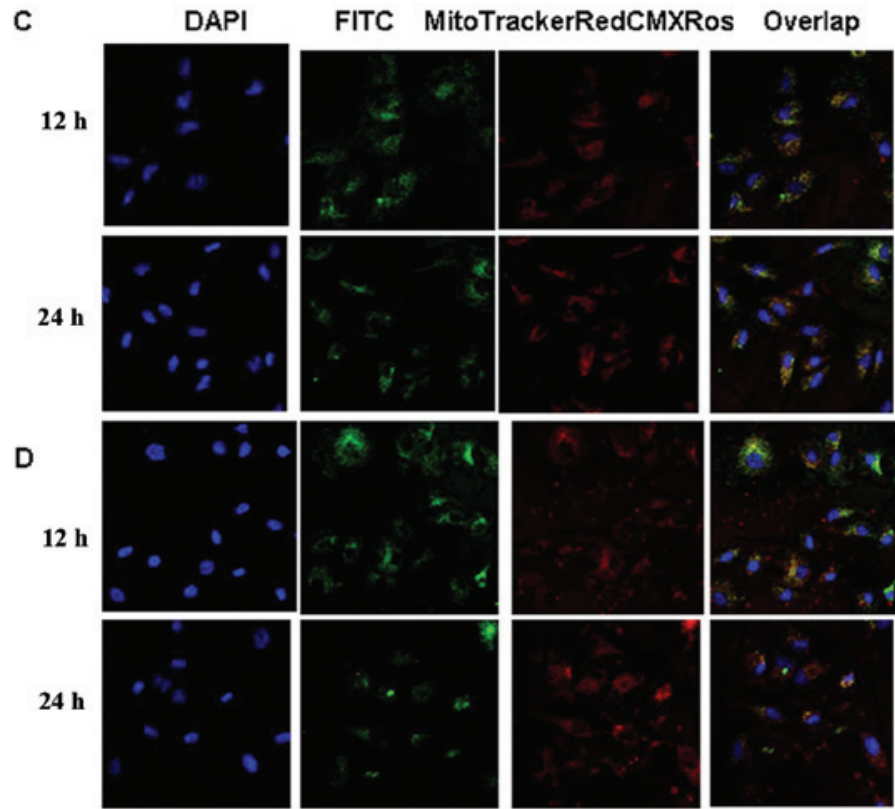

Figure 4. Effect of shear stress on the migration of Omi/HtrA2 protein. HUVECs were subjected to shear stress or incubated in a static condition for $12 \mathrm{~h}$ and $24 \mathrm{~h}$. (A and B) Western blot analysis was then performed to measure the level of Omi/HtrA2 protein. The mitochondrial and cytoplasmic proteins were separated. (A) Omi/HtrA2 protein was expressed mainly in the mitochondria following exposure to 15 dyne $/ \mathrm{cm}^{2}$ shear stress for $12 \mathrm{~h}$ and a similar result was observed at (B) $24 \mathrm{~h}$. Immunofluorescent staining of Omi/HtrA2 in HUVECs treated with or without exposure to 15 dyne/ $\mathrm{cm}^{2}$ shear stress for $12 \mathrm{~h}$ and $24 \mathrm{~h}$. (C) Cells cultured in $2 \%$ FBS DMEM medium were used as the static control group. (D) Cells treated with 15 dyne $/ \mathrm{cm}^{2}$ shear stress. Omi/HtrA2 protein is expressed in the cytoplasm (green) and mitochondria (red). This result is representative of three independent experiments. HUVECs, human umbilical vein endothelial cells.

To identify whether shear stress inhibited Omi/HtrA2 expression at the protein level, confluent HUVECs were subjected to laminar shear stress of $15 \mathrm{dyne} / \mathrm{cm}^{2}$ for varying periods of time. Reduced levels of Omi/HtrA2 protein were detected at 12, 18 and $24 \mathrm{~h}$, as analyzed by western blot analysis. Densitometry analysis revealed that exposure to shear stress decreased the amount of Omi/HtrA2 protein at 12, 18 and $24 \mathrm{~h}$ (Fig. 3D), compared with the levels in 
HUVECs kept in a static condition. Total protein expression of the control group, which was not exposed to shear stress, gradually increased (Fig. 3C). Total protein expression of the experimental group, which was exposed to $15 \mathrm{dyne} / \mathrm{cm}^{2}$ of shear stress, significantly decreased from $12 \mathrm{~h}$ to $24 \mathrm{~h}$ and was the lowest at $24 \mathrm{~h}$ (Fig. 3E).

Omi/HtrA2 protein migration. To examine whether the $\mathrm{Omi} / \mathrm{Htr} \mathrm{A} 2$ protein migrated between the cytoplasm and mitochondria following induction of apoptosis and protection by shear stress, we separated the total protein into cytoplasmic and mitochondrial fractions. We then used western blot analysis and immunofluorescence to analyze the results.

Mitochondrial and cytoplasmic proteins were separated using the isolation kit (Applygen, Beijing, China), and the Omi/HtrA2 protein levels were measured by western blot analysis. As shown in Fig. 4A and B, the mitochondrial protein was more weakly expressed than the cytoplasmic protein in the static control group. However, in the shear stress group, the mitochondrial protein was more strongly expressed than the cytoplasmic protein; this was more evident at $24 \mathrm{~h}$. In the static control group, mitochondrial protein was expressed more weakly, but cytoplasmic protein was expressed more strongly; consistent with the $12 \mathrm{~h}$ result. In the shear stress group, the mitochondrial protein is expressed even more strongly and the cytoplasmic protein is expressed even more strongly.

For the immunofluorescence analysis, we labeled nuclei with fluorescent DAPI (blue), Omi/HtrA2 with FITC (green), and mitochondria with MitoTracker Red CMXRos (red). Based on analysis of the images detected by fluorescent confocal microscopy (Fig. 4C and D), Omi/HtrA2 protein was gradually released into the cytoplasm from the mitochondria in the static control group following induction due to lack of bFGF and 2\% FBS DMEM medium for 12 and $24 \mathrm{~h}$ (Fig 4C). In the shear stress group (Fig. 4D), the Omi/HtrA2 protein was expressed mainly in the mitochondria following shear stress for 12 and $24 \mathrm{~h}$, consistent with the western blot analysis result. The results indicate that the apoptotic signal induced the HUVECs to express greater Omi/HtrA2 in the absence of the protective effect of shear stress.

\section{Discussion}

The present study demonstrates that Omi/HtrA2 mRNA and protein expression and release of Omi/HtrA2 from mitochondria into the cytoplasm in HUVECs was decreased by shear stress.

Atherosclerotic lesions are preferentially found in areas with low or turbulent shear stress, while areas exposed to steady laminar shear stress are protected $(3,4)$. A large body of evidence suggests that endothelial apoptosis contributes to the development of atherosclerotic lesions in areas of low or turbulent flow with prevalent occurrence of apoptosis in the downstream part of the plaque $(4,5)$. In vitro studies have demonstrated that laminar shear stress at physiological levels is sufficient to protect HUVECs from apoptotic cell death $(13,14)$. Several mechanisms have been proposed to account for the anti-apoptotic effects of laminar shear stress, including upregulation of anti-apoptotic Bcl-XL (14), IAPs $(6,7)$ and activation of Akt kinase (15). According to an earlier study, the intrinsic apoptotic pathway is involved after ECs receive apoptotic stimuli. In this pathway, the outer membrane of the mitochondria becomes permeable to cytochrome C. Once released to the cytosol, cytochrome $\mathrm{C}$ binds to Apaf-1 at a ratio of 2:1 forming an oligomeric Apaf-1/cytochrome $\mathrm{C}$ complex in the presence of dATP or ATP. This oligomerized Apaf-1/ cytochrome $\mathrm{C}$ complex then recruits the initiator caspase of this pathway, pro-caspase-9, and induces its autoactivation and the subsequent activation of caspase-3 and 7 (16). The first family of endogenous cellular inhibitors of caspases to be identified in mammals was the IAP family; these have been characterized over the past few years. The diversity of triggers against which IAP suppresses apoptosis is greater than that observed for any family of apoptosis inhibitors, including the Bcl-2 family. The central mechanisms of IAP apoptotic suppression are direct caspase and procaspase (caspases 3, 7 and 9) inhibition. Omi/HtrA2 is a mammalian serine protease that resides in the mitochondria of healthy cells. Omi/HtrA2 is the antagonist of IAP and is a nuclear-encoded mitochondrial protein. Following apoptotic stimulation, the signal sequence of Omi/HtrA2 in the mitochondria is cleaved, resulting in new apoptotic activity of the N-terminus. When Omi/HtrA2 is released into the cytoplasm, it binds and antagonizes the BIR actions of IAPs and releases the caspases binding to the BIR; thus, the caspases induce apoptosis (17). Omi/HtrA2 also promotes the catalytic cleavage of IAPs leading to their irreversible inactivation and the progression of apoptosis (8). In HUVECs, Omi/HtrA2 plays the same pre-apoptotic role (11). Inhibition of Omi/HtrA2 by a pharmacological approach provided significant protection against caspase- 3 activation (8-11). Very little is known about the pathophysiological role of Omi/HtrA2 in ECs during shear stress.

In the present study, we revealed that laminar shear stress significantly reduced the Omi/HtrA2 mRNA level from 4 to $6 \mathrm{~h}$, and also decreased Omi/HtrA2 protein expression from 12 to $24 \mathrm{~h}$. Omi/HtrA2 normally resides in the mitochondria. Western blot analysis and immunofluorescence results indicated that shear stress inhibited $\mathrm{Omi} / \mathrm{HtrA} 2$ release from the mitochondria to the cytoplasm. Our results also demonstrated that in the static control group, Omi/HtrA2 protein moved from the mitochondria to the cytoplasm due to lack of bFGF and 2\% FBS DMEM in the medium for 12 and 24 h.In addition, the apoptotic signal led the HUVECs to express even more Omi/HtrA2 in the absence of protection by shear stress. The Omi/HtrA2 protein was mainly expressed in the mitochondria, compared with in the cytoplasm following shear stress for 12 and $24 \mathrm{~h}$. The results suggested that shear stress inhibited Omi/HtrA2 release from the mitochondria to the cytoplasm, and reduced the expression of Omi/HtrA2.

Our findings suggest that inhibition of Omi/HtrA2 release into the cytoplasm is important for the protection of ECs from apoptosis. In our previous study, we demonstrated that HIAP-2 and XIAP mRNA and protein are induced by laminar shear stress in ECs and that IAPs play important roles in protecting ECs from apoptosis (6,7). Since Omi/HtrA2 promotes apoptosis by inhibiting IAPs, it is reasonable to assume that the decreased expression of Omi/HtrA2 may play a role in ECs exposed to laminar shear stress. Further investigation is required to determine the direct effects of HtrA2/Omi on ECs exposed to laminar shear stress. 
In conclusion, the present study provides the first evidence that physiological levels of laminar shear stress reduce Omi/HtrA2 expression and release into the cytoplasm in HUVECs in vitro. These data provide an explanation for the inhibitory effect of shear stress on apoptosis. Further studies to define the upstream and downstream molecular events involved in shear stress-induced Omi/HtrA2 expression are required to deepen our understanding of the mechanisms by which shear stress regulates endothelial functions. Laminar shear stress-induced inhibition of Omi/HtrA 2 produced by ECs is likely to play an important role in the formation and development of atherosclerosis.

\section{Acknowledgements}

This study was supported by a grant from the National Natural Science Foundation (30670512).

\section{References}

1. Dimmeler S, Haendeler J, Rippman V, Nehls M and Zeiher AM: Shear stress inhibits apoptosis of human endothelial cells. FEBS Lett 399: 71-74, 1996.

2. Haga M, Chen A, Gortler D, Dardik A and Sumpio BE: Shear stress and cyclic strain may suppress apoptosis in endothelial cells by different pathways. Endothelium 10: 149-157, 2003.

3. Ku DN, Giddens DP, Zarins CK and Glagov S: Pulsatile flow and atherosclerosis in the human carotid bifurcation: positive correlation between plaque location and low oscillating shear stress. Arteriosclerosis 5: 293-302, 1985.

4. Asakura T and Karino T: Flow patterns and spatial distribution of atherosclerotic lesions in human coronary arteries. Circ Res 66: 1045-1066, 1990.

5. Kaiser D, Freyberg MA and Friedl P: Lack of hemodynamic forces triggers apoptosis in vascular endothelial cells. Biochem Biophys Res Commun 231: 586-590, 1997.

6. Jin X, Mitsumata M, Yamane T and Yoshida Y: Induction of human inhibitor of apoptosis protein-2 by shear stress in endothelial cells. FEBS Letters 529: 286-292, 2002.
7. Jin X, Shu Q, Ling GL and Wang HL: X-linked inhibitor of apoptosis protein is regulated by laminar shear stress in cultured endothelial cells. Chin J Pharmacol Toxicol 17: 328-332, 2003.

8. Suzuki Y, Imai Y, Nakayama H, Takahashi K, Takio K and Takahashi R: A serine protease, HtrA2, is released from the mitochondria and interacts with XIAP, inducing cell death. Mol Cell 8: 613-621, 2001.

9. Soustiel JF and Larisch S: Mitochondrial damage: A target for new therapeutic horizons. Neurotherapeutics 7: 13-21, 2010.

10. Pruefer FG, Lizarraga F, Maldonado V and Melendez-Zajgla J: Participation of Omi/Htra2 serine-protease activity in the apoptosis induced by cisplatin on SW480 colon cancer cells. J Chemother 20: 348-354, 2008.

11. Liu QB, Liu LL, Lu YM, Tao RR, Huang JY, Han F and Lou YJ: The induction of reactive oxygen species and loss of mitochondrial $\mathrm{Omi} / \mathrm{HtrA} 2$ is associated with S-nitrosoglutathione-induced apoptosis in human endothelial cells. Toxicol Appl Pharmacol 244: 374-384, 2010.

12. Kim J, Kim DS, Park MJ, Cho HJ, Zervos AS, Bonventre JV and Park KM: Omi/HtrA2 protease is associated with tubular cell apoptosis and fibrosis induced by unilateral ureteral obstruction. Am J Physiol Renal Physiol 298: F1332-F1340, 2010.

13. Tricot O, Mallat Z, Heymes C, Belmin J, Leseche G and Tedgui A: Relation between endothelial cell apoptosis and blood flow direction in human atherosclerotic plaques. Circulation 101: 2450-2453, 2000.

14. Bartling B, Tostlebe H, Darmer D, Holtz J, Silber RE and Morawietz H: Shear stress dependent expression of apoptosis regulating genes in endothelial cells. Biochem Biophys Res Commun 278: 740-746, 2000.

15. Dimmeler S, Assmus B, Hermann C, Haendeler J and Zeiher AM: Fluid shear stress stimulates phosphorylation of Akt in human endothelial cells: involvement in suppression of apoptosis. Circ Res 83: 334-341, 1998

16. Li P, Nijhawan D, Budihardjo I, et al: Cytochrome C and dATP dependent formation of Apaf-1/ caspase-9 complex initiates an apoptotic protease cascade. Cell 91: 479-489, 1997.

17. Hegde R, Srinivasula SM, Zhang Z, et al: Identification of Omi/ HtrA2 as a mitochondrial apoptotic serine protease that disrupts inhibitor of apoptosis protein-caspase interaction. J Biol Chem 277: 432-438, 2002. 